\title{
On decoupling of linear time delay systems by generalized output feedback
}

\author{
Rabah Rabah Michel Malabre \\ Institut de Recherche en Communications et Cybernétique de Nantes \\ 1, rue de la Noë, B. P. 92101 \\ F-44321 Nantes Cedex 03, France \\ E-mail:\{rabah, malabredirccyn.ec-nantes.fr\}
}

\begin{abstract}
We consider the row-by-row decoupling problem for linear delay systems by output feedback. The characterization of the solvability of this problem is given in terms of some easily checkable structural conditions. The main contribution is, in particular, to use generalized output feedback laws which may incorporate derivatives of the delayed new reference.
\end{abstract}

Keywords: Linear Delay Systems, Row-by-row Decoupling, Structure at Infinity, Output feedback.

\section{Introduction}

Several authors have considered the row-by-row decoupling problem for delay systems. In [8] an algebraic solution was given which extends the classical result of Falb and Wolovich (1967). The condition of the non-anticipativity of the state feedback law was studied in [7]. A more general framework which includes delay systems was given by [1]. The structural approach was developed in [6] and partial characterization of the solvability of the given problem was discussed. The present authors gave [5] a more general solution which uses generalized state feedback, i.e. feedback which may include the derivatives of the delayed new reference. These derivatives in the control law occur when the decoupling precompensator is weak proper but not strong proper (see Definition 2.2). Results in [5] are however restricted to the state feedback case, which, for effective implementation, usualy requires the use of an observer.

In [10], was given a nice characterization of the row-by-row decoupling using output feedback. This approach allows to use only the transfer function matrix in the description of the solution and of the feedback, giving rise to solutions which are more robust than those based on state space methods. The state of the system is not needed and observers are not required. Our purpose is to extend the result of [10] to delay systems and to extend our result using generalized feedback. Namely, the aim of the paper is to characterize the row-by-row decoupling problem by generalized output feedback. As in [10], the characterization and the design of the decoupling feedback are given in terms of transfer function matrix. 


\subsection{System description}

We consider linear time-invariant systems with delays described by:

$$
\left\{\begin{array}{l}
\dot{x}(t)=A_{0} x(t)+A_{1} x(t-1)+B_{0} u(t) \\
y(t)=C_{0} x(t)
\end{array}\right.
$$

where $x(t) \in \mathbb{R}^{n}$ is the state, $u(t) \in \mathbb{R}^{m}$ is the control input, $y(t) \in \mathbb{R}^{m}$ is the output to be controlled. Without loss of generality, we can assume that $B_{0}$ is of full column rank. In order to simplify the notation and some computations, we limit ourselves to systems with a single delay in the state. All results and considerations given here remain valid for systems with several commensurate delays in the state. The transfer function matrix of the system (1) is

$$
T\left(s, e^{-s}\right)=C_{0}\left(s I-A_{0}-A_{1} e^{-s}\right)^{-1} B_{0}
$$

and may be expanded into two different ways, namely as a power series expansion, either in the variable $e^{-s}$ (with coefficients function of $s$ ) or in the variable $s$ (with coefficients function of $e^{-s}$ ). Both expansions are given using the matrices introduced by Kirillova and Churakova and compared with other tools in [9]:

$$
\begin{aligned}
& Q_{i}(j)=A_{0} Q_{i-1}(j)+A_{1} Q_{i-1}(j-1), \\
& Q_{0}(0)=I, Q_{i}(j)=0, i<0 \text { or } j<0 .
\end{aligned}
$$

The first expansion is

$$
T\left(s, e^{-s}\right)=\sum_{j=0}^{\infty} \sum_{i=0}^{\infty} C_{0} Q_{i}(j) B_{0} s^{-i-1} e^{-j s},
$$

The other expression, which will be used in this paper, is the following one

$$
T\left(s, e^{-s}\right)=\sum_{i=0}^{\infty} \sum_{j=0}^{i} C_{0} Q_{i}(j) B_{0} e^{-j s} s^{-i-1} .
$$

These expressions may be obtained by a simple calculation using the relations (2), see [6] and [9].

\subsection{Problem formulation}

We shall consider decoupling of systems like (1).

The "open-loop" definition of decoupling is the following: Find a precompensator $K\left(s, e^{-s}\right)$ and non identically zero scalar transfer functions $h_{i}\left(s, e^{-s}\right), i=1, \ldots, m$, such that

$$
T\left(s, e^{-s}\right) K\left(s, e^{-s}\right)=\operatorname{diag}\left\{h_{1}\left(s, e^{-s}\right), \ldots, h_{m}\left(s, e^{-s}\right)\right\} .
$$

We are interested in output feedback implementations of such decoupling precompensators, when they exist, and we want to connect the properties of $K\left(s, e^{-s}\right)$ which make it realizable in an output feedback form and the type of, more or less restricted, output feedback laws which may be used.

We have previously shown [3] (see also [6]) that any decoupling solution $K\left(s, e^{-s}\right)$ belonging to some particular class of precompensators, called strong biproper (see Section 2), is equivalent to a static state feedback control law of the type: $u(s)=F\left(e^{-s}\right) x(s)+G\left(e^{-s}\right) v(s)$, where $F\left(e^{-s}\right)$ and $G\left(e^{-s}\right)$ are proper matrices with respect to the variable $e^{s}$. In [5] we considered a broader class of decoupling precompensators, called weak biproper (see Section 2) and we showed their equivalence with generalized static state feedback control laws of the type:

$$
u(s)=F\left(e^{-s}\right) x(s)+G\left(s, e^{-s}\right) v(s),
$$


where $F\left(e^{-s}\right)$ and $G\left(s, e^{-s}\right)$ may be decomposed as follows

$$
\begin{aligned}
F\left(e^{-s}\right) & =F_{0}+F_{1} e^{-s}+\cdots, \\
G\left(s, e^{-s}\right) & =G_{0}+G_{1}(s) e^{-s}+\cdots,
\end{aligned}
$$

with possible polynomial matrices $G_{i}(s), i \geq 1, G_{0}$ and $F_{i}, i \in \mathbb{N}$ are constant matrices. Note that $F\left(e^{-s}\right)$ and $G\left(s, e^{-s}\right)$ are rational with respect to the corresponding arguments and this means that we need in fact a finite number of delays in the feedback. This amounts also to accepting in the control law some delayed derivatives of the new reference input $v(t)$ and allows to look for more general solutions, assuming that the reference input $v(t)$ is smooth enough for all its involved derivatives to exist.

The aim of the present paper is to extend some above mentioned results to the problem of decoupling by output feedback.

\section{Preliminaries}

In this section we recall classical results for systems without delays and recent results for decoupling by static state feedback for system with delays. First of all we give the notion of properness for linear systems and its extensions to linear time delay systems.

Definition 2.1 A rational complex valued function $f(s)$ is called proper if $\lim f(s)$ is finite when $|s| \rightarrow \infty$. It is called strictly proper if this limit is 0 . It is called biproper if this limit is invertible.

For rational functions this notion may be described by the degrees of the numerator and denominator. For systems with delay, the transfer function contains $e^{-s}$ which is not rational, we need then other notions of properness.

Definition 2.2 A complex valued function $f(s)$ is called weak proper if $\lim f(s)$ is finite when $s \in \mathbb{R}$ tends to $\infty$. It is called strictly weak proper if this limit is 0 . A matrix $B(s)$ is weak biproper if it is weak proper and if this limit is invertible. Weak proper is replaced by strong proper if the same occurs when $s \in \mathbb{C}$ and $\Re \mathrm{e}(s) \rightarrow \infty$.

It is obvious that strong properness implies weak properness. If the function is analytical at infinity all notions coincide, because the limits at infinity are the same. The strong properness is the natural extension of the classical notion, but does not give a well defined structure at infinity. The weak properness allows to give a good canonical form at infinity (see [5] and references given there).

In the sequel we shall use the different notions of properness given in Definitions 2.1 and 2.2: proper, weak proper and strong proper.

Let us recall the result concerning the decoupling by output feedback for linear systems without delay.

Theorem 2.3 [10] Let us consider the linear system

$$
\left\{\begin{array}{l}
\dot{x}(t)=A x(t)+B u(t) \\
y(t)=C x(t)
\end{array}\right.
$$

with square transfer function matrix $T(s)=C(s I-A)^{-1} B$. The following propositions are equivalent:

1. There exist a feedback law $u=H y+G v$ and non zero scalar transfer functions $h_{i}(s), i=$ $1, \ldots$, m such that

$$
C(s I-A-B H C)^{-1} B G=\operatorname{diag}\left\{h_{1}(s), \ldots, h_{m}(s)\right\}
$$


2. (a) The so-called Falb-Wolovich matrix

$$
D=\left[\begin{array}{c}
c_{1} A^{n_{1}-1} B \\
\vdots \\
c_{m} A^{n_{m}-1} B
\end{array}\right],
$$

is invertible. The integer $n_{i}, i=1, \ldots, m$ is the order of the zero at infinity of each row subsystem: $c_{i} A^{n_{i}-1} B \neq 0$ and $c_{i} A^{j} B=0$ for $j<n_{i}-1$.

(b) The off-diagonal elements of $D T(s)^{-1}$ are scalars (independent of $s$ ).

Let us note that if the state space representation is not known, then the matrix $D$ may be defined by

$$
T(s)=\Delta(s)^{-1}(D+W(s)),
$$

where $\Delta(s)=\operatorname{diag}\left\{s^{n_{1}}, \ldots, s^{n_{m}}\right\}, n_{i}$ the order of the zero at infinity of each row $i$.

For the delay system we need the following characterization of the decoupling problem by (generalized) state feedback.

Theorem 2.4 [5] The following propositions are equivalent:

1. The row-by-row decoupling problem for the delay system (1) is solvable by a weak biproper precompensator $K\left(s, e^{-s}\right)$ :

$$
T\left(s, e^{-s}\right) K\left(s, e^{-s}\right)=\operatorname{diag}\left\{h_{1}\left(s, e^{-s}\right), \ldots, h_{m}\left(s, e^{-s}\right)\right\} .
$$

2. The global and the row by row weak structures at infinity are equal:

$$
\Sigma_{\infty}^{w}\left(C_{0}, A_{0}, A_{1}, B_{0}\right)=\left[\begin{array}{c}
\Sigma_{\infty}^{w}\left(c_{1}, A_{0}, A_{1}, B_{0}\right) \\
\vdots \\
\Sigma_{\infty}^{w}\left(c_{m}, A_{0}, A_{1}, B_{0}\right)
\end{array}\right],
$$

where $c_{i}$ 's are the rows of the matrix $C_{0}$.

3. The generalized Falb-Wolovich matrix:

$$
D_{0}=\left[\begin{array}{c}
c_{1} Q_{n_{1}-1}\left(k_{1}\right) B_{0} \\
\vdots \\
c_{m} Q_{n_{m}-1}\left(k_{m}\right) B_{0}
\end{array}\right],
$$

is invertible, where for each row $i$ the integers $n_{i}$ and $k_{i}$ are such that: $c_{i} Q_{n_{i}-1}\left(k_{i}\right) B_{0} \neq 0$ and $c_{i} Q_{l}(j) B_{0}=0$ for $l<n_{i}-1$ and $j<k_{i}$.

4. The decoupling problem is solvable by generalized static state feedback

$$
u=F\left(e^{-s}\right) x+G\left(s, e^{-s}\right) v,
$$

where $F\left(e^{-s}\right)$ is strong proper and $G\left(s, e^{-s}\right)$ weak biproper.

Let us denote by $\Delta\left(s, e^{-s}\right)$ the matrix

$$
\Delta\left(s, e^{-s}\right)=\operatorname{diag}\left\{s^{n_{1}} e^{k_{1} s}, \ldots, s^{n_{m}} e^{k_{m} s}\right\} .
$$

Then the transfer function matrix of the delay system may be factorized as

$$
\begin{aligned}
T\left(s, e^{-s}\right) & =\Delta\left(s, e^{-s}\right)^{-1}\left(D\left(e^{-s}\right)+W\left(s, e^{-s}\right)\right), \\
& =\Delta\left(s, e^{-s}\right)^{-1}\left(D\left(e^{-s}\right)+W_{1}\left(s, e^{-s}\right)+W_{2}\left(s, e^{-s}\right)\right),
\end{aligned}
$$

where $D\left(e^{-s}\right)=D_{0}+D_{1} e^{-s}+\ldots, W_{1}\left(s, e^{-s}\right)$ and $W_{2}\left(s, e^{-s}\right)$ being respectively the strictly strong proper and the strictly weak proper parts in this unique decomposition (see [5]). 
Theorem 2.5 If the delay system (1) is decouplable by static state feedback then $D_{0}$ is invertible and $D\left(e^{-s}\right)$ is strong biproper.

PRoOF: If the system (1) is decouplable by static state feedback then it is decouplable by generalized state feedback and this implies that $D_{0}$ is invertible. This means that $D\left(e^{-s}\right)$ is biproper in the strong sense.

Theorem 2.6 If $D_{0}$ is invertible and $W_{2}\left(s, e^{-s}\right)=0$, then (1) is decouplable by static state feedback.

Proof: If the condition of the theorem are satisfied then $K\left(s, e^{-s}\right)=\left(D\left(e^{-s}\right)+W_{1}\left(s, e^{-s}\right)\right)^{-1}$ is a strong biproper decoupling compensator. According to [5], it may be realizable by static state feedback.

Note that the converse of this theorem is not true. For example, consider the decoupled system

$$
T\left(s, e^{-s}\right)=\left[\begin{array}{cc}
s^{-2}+s^{-1} e^{-s} & 0 \\
0 & s^{-1}
\end{array}\right] .
$$

For this system, $D_{0}=I, W_{2}\left(s, e^{-s}\right) \neq 0$ and $W_{1}\left(s, e^{-s}\right)=0$.

\section{Decoupling by output feedback}

In order to simplify the notation, we are omitting sometimes the arguments of the corresponding function. The arguments are precised when it stands necessary.

We consider first the problem of decoupling by static output feedback of the form

$$
u(s)=H\left(e^{-s}\right) y(s)+G\left(e^{-s}\right) v(s),
$$

with $H\left(e^{-s}\right)$ and $G\left(e^{-s}\right)$ proper in $e^{s}$. If the feedback is given by (7), then the corresponding input-output relation may be written as

$$
y=T[I-H T]^{-1} G v=\left[T^{-1}-H\right]^{-1} G v .
$$

Theorem 3.1 The system (1) is decouplable by static output feedback if and only if i) It is decouplable by static state feedback

ii) The off-diagonal elements of the matrix

$$
D\left(e^{-s}\right) T\left(s, e^{-s}\right)^{-1}
$$

are proper in $e^{s}$ and do not depend explicitly on $s$.

The condition ii) may be precised as follows. If we denote by $\alpha_{i j}\left(s, e^{-s}\right)$ the off-diagonal elements, then ii) is equivalent to: $\alpha_{i j}\left(s, e^{-s}\right)=a_{0}+a_{1} e^{-s}+a_{2} e^{-2 s}+\ldots, i \neq j$, where $a_{k}$ are constants depending on $i$ and $j$.

PROOF: The proof is given in the Annex.

\section{Decoupling by generalized output feedback}

In many cases, static state feedback is not sufficient to decouple linear system with delay even when there exists a decoupling precompensator (see the Example in this section). For the output feedback decoupling the same situation occurs. Then we need, as for the state feedback law (see [5]), to extend the condition of realization. This is the aim of this section. Let us give a preliminary result. 
Lemma 4.1 Let $T\left(s, e^{-s}\right)$ be decomposed as in (6). Then

$$
T_{1} \stackrel{\text { def }}{=} \Delta^{-1}\left(D+W_{1}\right)=T\left[I+\left(D+W_{1}\right)^{-1} W_{2}\right]^{-1} .
$$

Proof: This may be obtained by a simple calculation.

Lemma 4.2 The system $T\left(s, e^{-s}\right)$ is decouplable by generalized output feedback if and only if the system $T_{1}\left(s, e^{-s}\right)$ is decouplable by static output feedback.

Proof: The proof is given in the Annex.

Theorem 4.3 The system (1) is decouplable by generalized output feedback if and only if i) $D_{0}$ is invertible (it is decouplable by generalized state feedback).

ii) The off-diagonal elements of the matrix $D\left(e^{-s}\right) T_{1}\left(s, e^{-s}\right)^{-1}$ are proper in $e^{s}$ and do not depend explicitly on $s$.

Proof: Suppose that the condition of the theorem are satisfied. i) implies that $D\left(e^{-s}\right)$ is strong biproper (Theorem 2.5) and then $T_{1}\left(s, e^{-s}\right)=\Delta\left(s, e^{-s}\right)^{-1}\left(D\left(e^{-s}\right)+W_{1}\left(s, e^{-s}\right)\right)$ is decouplable by static state feedback (Theorem 2.6 ). Then the condition ii) and Theorem 3.1 give that $T_{1}\left(s, e^{-s}\right)$ is decouplable by static output feedback. This and Lemma 4.2 give the result. Conversely, if the system $T\left(s, e^{-s}\right)$ is decouplable by generalized output feedback, then the condition i) is obviously satisfied and the condition ii) is the consequence of Lemma 4.2.

\subsection{Procedure}

As the main calculations are given in the proofs, we give here a short procedure to design the decoupling feedback if applicable. Let be given a transfer function matrix $T\left(s, e^{-s}\right)$.

1. For each row $i=1, \ldots, m$ consider the first non zero moment, say $s^{-n_{i}} e^{-k_{i} s}$. The corresponding row-vectors coefficients form the matrix $D_{0}$. If this matrix is not invertible, the system is not decouplable. If it is invertible, the system is decouplable by generalized state feedback.

2. Suppose that $D_{0}$ is invertible. Consider now $\Delta\left(s, e^{-s}\right)=\operatorname{diag}\left\{s^{n_{1}} e^{k_{1} s}, \ldots, s^{n_{m}} e^{k_{m} s}\right\}$. The matrix $\Delta\left(s, e^{-s}\right) T\left(s, e^{-s}\right)$ can be decomposed as follows (the decomposition is unique):

$$
\Delta\left(s, e^{-s}\right) T\left(s, e^{-s}\right)=D\left(e^{-s}\right)+W_{1}\left(s, e^{-s}\right)+W_{2}\left(s, e^{-s}\right),
$$

where $D$ is strong biproper and does not depend explicitly on $s, W_{1}$ is strictly strong proper and $W_{2}$ strictly weak proper.

3. Let us put $T_{1}=\Delta^{-1}\left[D+W_{1}\right]$. If the off-diagonal elements of $D T^{-1}$ do not depend on $s$ and are proper in $e^{s}$ (Theorem 4.3), then the system is decouplable by generalized output feedback, then go to 4 . Otherwise the system is not decouplable by output feedback.

4. Let us now give a feedback law solution. Let $\delta\left(s, e^{-s}\right)$ be the diagonal of $D T^{-1}$. One can take then

$$
H\left(e^{-s}\right)=D\left(e^{-s}\right)^{-1}\left[D\left(e^{-s}\right) T^{-1}\left(s, e^{-s}\right)-\delta\left(s, e^{-s}\right)\right]
$$

Let $\Lambda\left(e^{-s}\right)$ be any diagonal matrix, proper in $e^{s}$ and $G_{1}\left(e^{-s}\right)=D\left(e^{-s}\right) \Lambda\left(e^{-s}\right)$. Putting

$$
G=\left[I+\left(D+W_{1}\right)^{-1} W_{2}(I-H T)^{-1}\right]^{-1} G_{1}
$$

we get the decoupling feedback law $u=H\left(e^{-s}\right) y+G\left(s, e^{-s}\right) v$. 


\subsection{Example}

Let us consider the system

$$
T\left(s, e^{-s}\right)=\left[\begin{array}{cc}
s^{-3} & \left(s^{-4}+s^{-2}\right) e^{-s} \\
0 & s^{-1}
\end{array}\right]
$$

The coupling between the second input and the first output equals $\left(s^{-4}+s^{-2}\right) e^{-s}$. If we use some classical approximation, namely the Padé approximation, the obtained rational system (without delay) is not decouplable. Indeed, the Padé approximation of $e^{-s}$ is a biproper function and then the obtained system is equivalent (for the problem of decoupling) to the system

$$
\left[\begin{array}{cc}
s^{-3} & \left(s^{-4}+s^{-2}\right) \\
0 & s^{-1}
\end{array}\right]
$$

The Falb-Wolovich matrix $D$ of this system is

$$
D=\left[\begin{array}{ll}
0 & 1 \\
0 & 1
\end{array}\right]
$$

and then the system is not decouplable (see Theorem 2.3).

It can be easily checked that there is no static state feedback law which decouples this system (9). However, $K\left(s, e^{-s}\right)=\left[\begin{array}{cc}1 & -\left(s^{-1}+s\right) e^{-s} \\ 0 & 1\end{array}\right]$ is a decoupling precompensator. This precompensator is weak but not strong biproper, which means that it cannot be realizable by static state feedback. Here, in this particular case, $D_{0}=D\left(e^{-s}\right)=I$ and then the system is decouplable by generalized state feedback (see Theorem 2.4). We have $W_{1}\left(s, e^{-s}\right)=\left[\begin{array}{cc}0 & s^{-1} e^{-s} \\ 0 & 0\end{array}\right]$ and then

$$
T_{1}\left(s, e^{-s}\right)^{-1}=\left[\begin{array}{cc}
s^{3} & -e^{-s} \\
0 & s
\end{array}\right] .
$$

Then $D\left(e^{-s}\right) T_{1}\left(s, e^{-s}\right)^{-1}$ verifies the condition of the Theorem 4.3 Therefore system (9) is decouplable by generalized output feedback. The output feedback is $u=H y+G v$, with

$$
H\left(e^{-s}\right)=\left[\begin{array}{cc}
0 & -e^{-s} \\
0 & 0
\end{array}\right], \quad G\left(s, e^{-s}\right)=\left[\begin{array}{cc}
1 & -s e^{-s} \\
0 & 1
\end{array}\right]
$$

This generalized output feedback law gives a decoupled system with transfer function matrix: $\operatorname{diag}\left\{s^{-3}, s^{-1}\right\}$.

\section{Conclusion}

Generalized output feedback law is used to decouple linear systems with delays. The conditions given here are easy to verify. The counterpart of the general framework is that we need the derivative of the delayed new control. This requires smoothness of the new control.

\section{Annex}

Recall that we are omitting arguments of some functions. They are precised when it stands necessary. 


\subsection{Proof of the Theorem 3.1}

Suppose that the conditions of the theorem are satisfied. Then (see [5]) $D\left(e^{-s}\right)$ is strongly biproper and so is $D\left(e^{-s}\right)^{-1}$. Let

$$
\delta\left(s, e^{-s}\right)=\left\{\delta_{1}\left(s, e^{-s}\right), \ldots, \delta_{m}\left(s, e^{-s}\right)\right\}
$$

be the diagonal of $D\left(e^{-s}\right) T\left(s, e^{-s}\right)^{-1}$. Then

$$
D\left(e^{-s}\right) T\left(s, e^{-s}\right)^{-1}-\delta\left(s, e^{-s}\right)
$$

is proper in $e^{s}$ and does not depend explicitly on $s$. Let us denote

$$
H \stackrel{\text { def }}{=} D^{-1}\left[D T^{-1}-\delta\right] .
$$

This gives

$$
D T^{-1}-D H=\delta,
$$

or

$$
\left[T^{-1}-H\right]^{-1} D^{-1}=\delta^{-1}
$$

Moreover, the diagonal matrix $\delta\left(s, e^{-s}\right)^{-1}$ is strictly strong proper, because $\delta\left(s, e^{-s}\right)$ is the diagonal of the matrix

$$
\left(I+W\left(s, e^{-s}\right) D\left(e^{-s}\right)^{-1}\right) \Delta\left(s, e^{-s}\right),
$$

according to the formulae (6). Let now $\Lambda\left(e^{-s}\right)$ be any diagonal matrix, proper in $e^{s}$. Then

$$
\delta^{-1} \Lambda=\left[T^{-1}-H\right]^{-1} D^{-1} \Lambda
$$

Putting $G\left(e^{-s}\right)=D\left(e^{-s}\right)^{-1} \Lambda\left(e^{-s}\right)$, the above relation means that the system is decouplable by the feedback

$$
u(s)=H\left(e^{-s}\right) y(s)+G\left(e^{-s}\right) v(s),
$$

according to the relation (7). The closed loop transfer function matrix is

$$
T_{d}\left(s, e^{-s}\right)=\delta\left(s, e^{-s}\right)^{-1} \Lambda\left(e^{-s}\right) .
$$

Conversely, suppose that the system is decouplable by the output feedback law (7). Then

$$
\left[T\left(s, e^{-s}\right)^{-1}-H\left(e^{-s}\right)\right]^{-1} G\left(e^{-s}\right)=T_{d}\left(s, e^{-s}\right)
$$

with diagonal matrix $T_{d}\left(s, e^{-s}\right)$. A simple calculation gives

$$
D T^{-1}=D G T_{d}^{-1}+D H .
$$

In order to show the condition ii) of the theorem, it is sufficient to show that $D\left(e^{-s}\right) G\left(e^{-s}\right)$ is a diagonal matrix.

Note that (see (6)) $\Delta\left(s, e^{-s}\right) T\left(s, e^{-s}\right)$ is a weak biproper matrix. As $T\left(s, e^{-s}\right)$ and $T_{d}\left(s, e^{-s}\right)$ differ by a weak biproper matrix (they are equivalent at infinity and have the same structure at infinity), then the diagonal $\Delta\left(s, e^{-s}\right) T_{d}\left(s, e^{-s}\right)$ is also weak biproper. Then

$$
B\left(s, e^{-s}\right)=T_{d}\left(s, e^{-s}\right)^{-1} \Delta\left(s, e^{-s}\right)^{-1}
$$

is a weak biproper diagonal matrix. From the decoupling condition we have

$$
T\left(s, e^{-s}\right)^{-1}=G\left(e^{-s}\right) T_{d}\left(s, e^{-s}\right)^{-1}+H\left(e^{-s}\right)
$$


and then

$$
T^{-1} \Delta^{-1}=G T_{d}^{-1} \Delta^{-1}+H \Delta^{-1} .
$$

From the expression (11) this equation may be written as

$$
T^{-1} \Delta^{-1}=G B \Delta \Delta^{-1}+H \Delta^{-1} .
$$

which gives

$$
T^{-1} \Delta^{-1}=G B+H \Delta^{-1} .
$$

On the other hand, from (6) we get

$$
T^{-1} \Delta^{-1}=(D+W)^{-1}
$$

From (12) and (13) we obtain

$$
(D+W)^{-1}=\quad G B+H \Delta^{-1} .
$$

By identification, we can obtain

$$
G\left(e^{-s}\right) B_{0}\left(e^{-s}\right)=D\left(e^{-s}\right)^{-1},
$$

where $B_{0}\left(e^{-s}\right)$ is the "constant" (according to the explicit dependence of $s$ ) part of the strong biproper matrix $B\left(s, e^{-s}\right)$. Let us precise that $B_{0}\left(e^{-s}\right)$ is diagonal because $B\left(s, e^{-s}\right)$ is. Putting $\Lambda\left(e^{-s}\right)=B_{0}^{-1}\left(e^{-s}\right)$ we obtain:

$$
D\left(e^{-s}\right) G\left(e^{-s}\right)=\Lambda\left(e^{-s}\right),
$$

which is a diagonal matrix, proper in $e^{s}$. Consider now the expression of $D\left(e^{-s}\right) T\left(s, e^{-s}\right)^{-1}$ given by (10). The fact that $D\left(e^{-s}\right) G\left(e^{-s}\right)=\Lambda\left(e^{-s}\right)$ is diagonal proves that the off-diagonal elements of $D\left(e^{-s}\right) T\left(s, e^{-s}\right)^{-1}$ are the off diagonal elements of $D\left(e^{-s}\right) H\left(e^{-s}\right)$. This gives ii).

\subsection{Proof of the Lemma 4.2}

As in both cases the system is decouplable by state feedback, $D\left(e^{-s}\right)$ is biproper in the strong sense (and then in the weak sense), which means that $\left(D+W_{1}\right)^{-1}$ is well defined for $s>s_{0}$. Let $H\left(e^{-s}\right)$ and $G_{1}\left(e^{-s}\right)$ be proper in $e^{s}$ matrices and let us denote $\Omega \stackrel{\text { def }}{=}\left(D+W_{1}\right)^{-1} W_{2}$. From Lemma 4.2 we have

$$
\begin{aligned}
T_{1}\left(I-H T_{1}\right)^{-1} G_{1} & \left.=T(I+\Omega)^{-1}\left[I-H T(I+\Omega)^{-1}\right]^{-1}\right) G_{1} \\
& =T\left[\left(I-H T(I+\Omega)^{-1}\right)(I+\Omega)\right]^{-1} G_{1} \\
& =T[I-H T+\Omega]^{-1} G_{1} \\
& =T(I-H T)^{-1}\left[I+\Omega(I-H T)^{-1}\right]^{-1} G_{1} .
\end{aligned}
$$

Let us now put $G=\left[I+\Omega(I-H T)^{-1}\right]^{-1} G_{1}$ and $G_{2}=G-G_{1}$. Then

$$
T_{1}\left(I-H T_{1}\right)^{-1} G_{1}=T(I-H T)^{-1} G,
$$

with $G=\left[I+\Omega(I-H T)^{-1}\right]^{-1} G_{1}=G_{1}+G_{2}$.

It is not difficult to see that $G_{2}$ is a function of two arguments $\left(s, e^{-s}\right)$, weak proper in $s$ if $G_{1}\left(e^{-s}\right)$ is strong biproper in $s$. Suppose now that $u(s)=H\left(e^{-s}\right) y(s)+G_{1}\left(e^{-s}\right) v(s)$ decouples the system $T_{1}\left(s, e^{-s}\right)$. This gives $T_{1}\left(I-H T_{1}\right)^{-1} G_{1}=T_{d}$, where a non singular $T_{d}\left(s, e^{-s}\right)$ is a diagonal matrix. Taking $G$ as indicated, we get

$$
T(I-H T)^{-1} G=T_{d} .
$$


Conversely, suppose that $T\left(s, e^{-s}\right)$ is decouplable by the generalized output feedback

$$
u(s)=H\left(e^{-s}\right) y(s)+G\left(s, e^{-s}\right) v(s),
$$

then $T(I-H T)^{-1} G=T_{d}$, with weak biproper $G\left(s, e^{-s}\right)$. The transfer function matrix $T_{d}\left(s, e^{-s}\right)$ may also be decomposed as $T\left(s, e^{-s}\right)$ in (6):

$$
T_{d}=\Delta^{-1}\left(D_{d}+W_{1 d}+W_{2 d}\right)
$$

with the same matrix $\Delta$ because of the equivalence at infinity, $W_{1 d}\left(s, e^{-s}\right)$ being strongly strictly proper and $W_{2 d}\left(s, e^{-s}\right)$ weakly strictly proper. This gives

$$
T(I-H T)^{-1} G-\Delta^{-1} W_{2 d}=\Delta^{-1}\left(D_{d}+W_{1 d}\right) \stackrel{\text { def }}{=} T_{1 d},
$$

with diagonal matrix $T_{1 d}$. This gives

$$
T_{1 d}=T(I-H T)^{-1}\left[G-(I-H T)\left(D+W_{1}+W_{2}\right)^{-1} W_{2 d}\right],
$$

where we used one more time the decomposition (6) for $T$. Let us denote

$$
\Gamma \stackrel{\text { def }}{=} G-(I-H T)\left(D+W_{1}+W_{2}\right)^{-1} W_{2 d}
$$

and $G_{1} \stackrel{\text { def }}{=}\left[I+\Omega(I-H T)^{-1}\right] \Gamma$. This gives

$$
T(I-H T)^{-1} \Gamma=T_{1 d}
$$

and

$$
T(I-H T)^{-1}\left[I+\Omega(I-H T)^{-1}\right]^{-1} G_{1}=T_{1 d} .
$$

Then using the same calculation as above we get

$$
T_{1}\left(I-H T_{1}\right)^{-1} G_{1}=T_{1 d} .
$$

which means that $T_{1}$ is decouplable by output feedback.

Let us now precise the structure of $G_{1}$. Multiplying by $\Delta\left(s, e^{-s}\right)$ both parts, we obtain

$$
\left(D+W_{1}\right)\left(I-H T_{1}\right)^{-1} G_{1}=D_{d}+W_{1 d},
$$

and by identification $D G_{1}=D_{d}$ and then

$$
G_{1}\left(e^{-s}\right)=D^{-1}\left(e^{-s}\right) D_{d}\left(e^{-s}\right) .
$$

Note that the fact that $G_{1}$ depends explicitly only on $e^{-s}$ may be obtained directly from the construction of $G_{1}$.

\section{References}

[1] Datta K. B., Hautus M. L. J. (1984). Decoupling of multivariable control systems over unique factorization domains. SIAM J. Control and Optimization, 22, pp. 28-39.

[2] Falb P. L., Wolovich W. A. (1967). Decoupling in the design and synthesis of multivariable control systems, I.E.E.E. Trans. Autom. Contr., AC-12, No. 12, 651-659.

[3] Rabah R., Malabre M. (1996). Structure at infinity for delay systems revisited. In: IMACS and IEEE-SMC Multiconference CESA'96, Symposium on Modelling, Analysis and Simulation, Lille, France, July 9-12, 87-90. 
[4] Rabah R., Malabre M. (1997). A note on decoupling for linear infinite dimensional systems.In: Proc. 4-th IFAC Conf. on Syst. Structure and Contr., Bucharest, Oct. 23-25, 7883.

[5] Rabah R., Malabre M. (1999). The structure at infinity of linear delay systems and the row-by-row decoupling problem. In: Proceedings of the 7th IEEE Mediterranean Conference on Control and Automation, Haifa, Israel, June 28-30, 1999, pp. 1845-1854.

[6] Sename O., Rabah R., Lafay J.-F. (1995), Decoupling without prediction of linear systems with delays: a structural approach. Syst. Contr. Letters 25, 387-395.

[7] Rekasius Z. V., Milzareck R. J. (1977), Decoupling without prediction of systems with delays. In Proc. Joint. Automat. Control Conf., San Francisco.

[8] Tzafestas S. G., Paraskevopoulos P. N. (1973). On the decoupling of multivariable control systems with time-delays, Int. J. Control, 17, pp. 405-415.

[9] A. C. Tsoi (1978). Recent advances in the algebraic system theory of delay differential equations. In: Recent theoretical developments in control, M. J. Gregson Ed., Academic Press, N. Y., pp. 67-127.

[10] Wolovich W. A. (1975). Ouput feedback decoupling. IEEE Transaction on Automatic Control, AC-20, pp. 148-151. 